\title{
Effects of Different Bandaging Methods for Treating Patients With Breast Cancer-Related Lymphedema
}

\author{
Se Hyun Oh, MD¹, Sung Hwan Ryu, MD', Ho Joong Jeong, MD, PhD ${ }^{1}$, \\ Jung Hyun Lee, MD, $\mathrm{PhD}^{2}$, Young-Joo Sim, MD, $\mathrm{PhD}^{1}$
}

Departments of ${ }^{1}$ Physical Medicine and Rehabilitation, ${ }^{2}$ Pediatrics, Kosin University Gospel Hospital, Busan, Korea

Objective To compare the treatment effects, satisfaction with the treatment, and performance improvement following bandage treatment using the spiral method and spica method for breast cancer-related lymphedema (BCRL).

Methods A prospective study with 46 patients with BCRL was conducted. All patients were divided into either the spiral or spica group for non-elastic bandage therapy and received the same treatment for 2 weeks, apart from the group-specific bandaging method used. For both groups, the Quality of Life Instrument score before treatment, changes in the volume of lymphedema limb and the Disability of the Arm, Shoulder, and Hand (DASH) score before and after treatment, and treatment satisfaction after treatment were compared. The Student t-test was used to compare the parameters between the two different bandage methods.

Results With respect to the treatment outcomes, total volume reduction and proximal part volume reduction after treatment were $98.0 \pm 158.3 \mathrm{~mL}$ and $56.0 \pm 65.4 \mathrm{~mL}$ in the spiral method group and $199.0 \pm 125.1 \mathrm{~mL}$ and $106.1 \pm 82.2$ $\mathrm{mL}$ in the spica method group, respectively. Therefore, the spica method group showed a significantly better improvement $(\mathrm{p}<0.05)$. The DASH score changes after treatment showed that the spiral group score increased by $3.8 \pm 5.4$ and the spica group score increased by $7.7 \pm 6.1$; thus, a significantly better improvement was noted in the spica group $(\mathrm{p}<0.05)$.

Conclusion The spica method indicated better volume reduction and DASH score improvement than the spiral method. Therefore, the spica method may be more effective for treating patients with BCRL.

Keywords Lymphedema, Breast cancer lymphedema, Bandages

Received April 26, 2019; Accepted July 4, 2019

Corresponding author: Young-Joo Sim

Department of Physical Medicine and Rehabilitation, Kosin University Gospel Hospital, 262 Gamcheon-ro, Seo-gu, Busan 49267, Korea. Tel: +82-51990-6261, Fax: +82-51-990-3181, E-mail: oggum@naver.com

ORCID: Se Hyun Oh (http://orcid.org/0000-0001-6754-8669); Sung Hwan Ryu (http://orcid.org/0000-0003-0440-6481); Ho Joong Jeong (http://orcid. org/0000-0002-0607-2799); Jung Hyun Lee (http://orcid.org/0000-0002-0496-9826); Young-Joo Sim (http://orcid.org/0000-0002-0640-8766).

(c) This is an open-access article distributed under the terms of the Creative Commons Attribution Non-Commercial License (http://creativecommons.org/ licenses/by-nc/4.0) which permits unrestricted noncommercial use, distribution, and reproduction in any medium, provided the original work is properly cited. Copyright $\odot 2019$ by Korean Academy of Rehabilitation Medicine 


\section{INTRODUCTION}

The desired outcome following treatment for lymphedema is reduction of swelling, not curing the underlying ailment. Several methods are commonly used to treat lymphedema, including manual lymphatic drainage (MLD), skin care, remedial exercise, compression bandages, compression garments, pneumatic compression, mercury compression, elevation, microwave, and laser therapies [1]. Complex decongestive therapy (CDT), which combines these various methods, was developed in Europe in the 1930s and is now recognized as an effective non-surgical treatment for lymphedema and is recommended by the International Society of Lymphology.

CDT involves education regarding skin management to prevent infection, MLD for fluid drainage, non-elastic bandage compression therapy for volume reduction, and remedial exercise [2]. Moreover, the use of compression bandages was previously reported to maintain the functional status of the bandaged upper extremity and to reduce swelling volume in the initial treatment stage of breast cancer-related lymphedema (BCRL) [3].

The bandages used provide minimal stretch and are mainly used to maintain the reduced volume following MLD. The bandage exerts light pressure during rest and high pressure during muscle contraction to prevent skin extension. The pressure between the muscles and the bandage facilitates passive lymphatic flow. The variable pressure across the bandaged skin when the muscle contracts is identical to the effect of massage, which increases lymph flow. The bandage should be worn as long as possible, including at night [4]. Recently, a variety of new products for compression therapy have been introduced to increase effectiveness and patient comfort.

As described above, lymphedema has a variety of treatment methods and each treatment has a therapeutic effect compared to no treatment $[5,6]$. However, a comparative study of the therapeutic effects of each treatment method is lacking and there is some disagreement on the results. For example, there have been many discussions about which components of the physical therapy program are most important and whether bandages are more effective in reducing edema than compression socks [7]. However, there have been no studies comparing different bandaging methods. The purpose of this study was to compare the treatment effects, treatment satisfaction, and performance improvement following bandage treatment using the spiral method and spica method for BCRL (Fig. 1).

\section{MATERIALS AND METHODS}

This was a prospectively designed study consisting of 46 patients with BCRL who were admitted to the Department of Rehabilitation Medicine of Kosin University Gospel Hospital, Korea for CDT. This study was approved by the Institutional Review Board of Kosin University College of Medicine (No. 201604-BM-008). And written consent was obtained for all participants.

Individuals meeting the following two criteria were included in the study: (1) patients with clinically unilateral upper limb edema after breast cancer surgery and
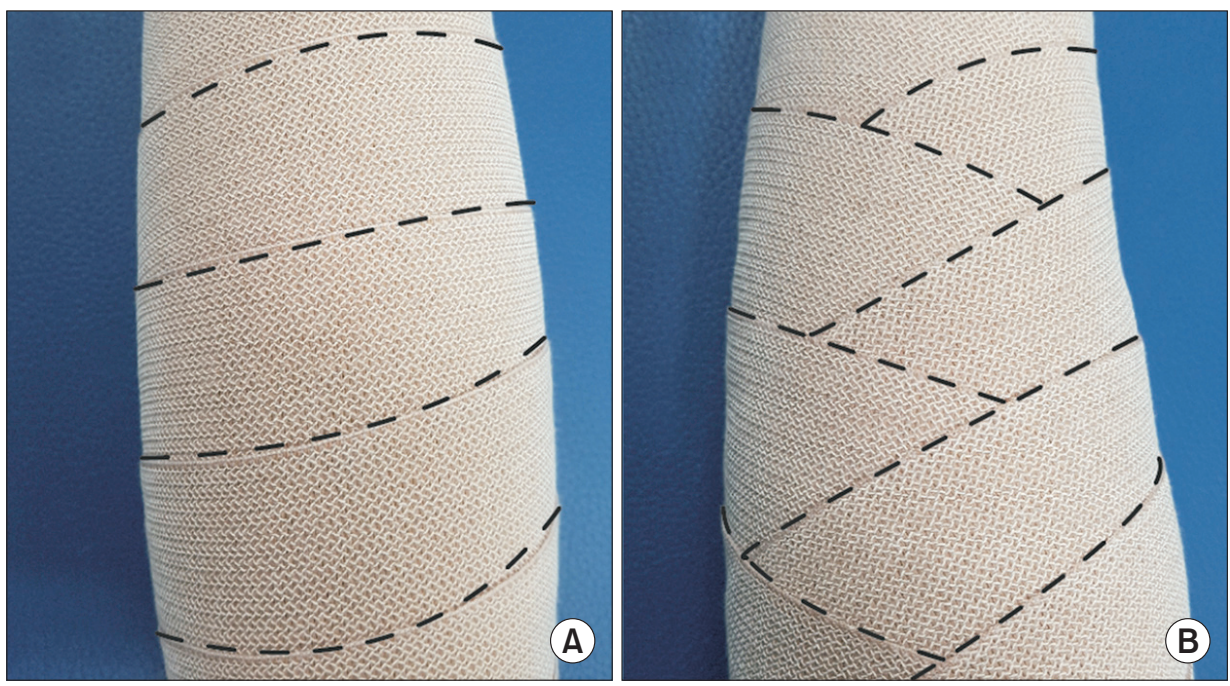

Fig. 1. Bandaging methods: (A) spiral method bandaging and (B) spica method bandaging. 
(2) patients diagnosed with lymphedema by lymphoscintigraphy and bioimpedance analysis (BIA). Patients were clinically diagnosed with lymphedema when the affected limb increased more than $2 \mathrm{~cm}$ in circumference or increased more than $200 \mathrm{~mL}$ in volume compared with the unaffected limb [8]. Lymphoscintigraphy confirmed lymphedema, in which the axillary lymph node uptake of the affected side was not observed till 3 hours or the flow was decreased. A mean limb index ratio (LIR) of 1.139 or more for the affected dominant arms and 1.066 or more for the affected non-dominant arms indicates a lymphedema diagnosis in patients with BIA [9].

Individuals meeting the following criteria were excluded: (1) patients with bilateral upper limb edema; (2) patients with edema due to impaired arterial or venous function; (3) patients with systemic edema due to an impaired heart, kidney, or liver function; (4) patients who were taking medication that could cause fluid retention and extremity swelling or those undergoing radiation or chemotherapy at the time of CDT; (5) patients who could not undergo CDT due to infection in the upper limb where the lymphedema was located; (6) patients who underwent CDT within the previous 3 months; (7) patients who were over 70 years old or who were unable to adhere to CDT guidelines due to cognitive impairment; and (8) patients with metastatic lymphedema.

All patients were divided into either the spiral group or the spica group for non-elastic bandage therapy and received the same treatment for 2 weeks with the exception of their group-specific bandaging method.

\section{General characteristics}

Patient age, weight, body mass index (BMI), breast cancer stage, the time from breast cancer operation to the onset of lymphedema, period of lymphedema, type of surgery, and lymph node surgery were assessed to compare characteristics between patient groups. The period of lymphedema was defined as the period from the time when the patient felt that the first swelling occurred to the start of CDT, and the above period was defined as the disease period. To quantify the effect of lymphedema on patients' daily lives, the Quality of Life Instrument (breast cancer patient version) was administered. The Quality of Life Instrument is an assessment tool containing 46 items designed to assess the quality of life (QOL) of breast cancer patients before, during, and after severe illness, pain, and surgery.

\section{Assessment}

The circumference and volume of both upper extremities were measured before CDT and 2 weeks after the CDT. The circumference of the arm was measured using 3 $\mathrm{cm}$ intervals to calculate the proximal and distal volume of the affected limb $[10,11]$. The total volume of the affected limb was measured using volumetry. Specifically, to determine the volume of each of the distal and proximal parts, an imaginary line connecting the olecranon with the medial and lateral epicondyle was defined as the anatomic baseline. The proximal part of the upper limb was defined as the area from the anatomic baseline to the axilla, and the distal part of the upper limb was defined as the area from the anatomic baseline to $6 \mathrm{~cm}$ below the ulnar styloid process.

We considered each segment as a truncated cone and calculated the segmental volume of each truncated cone. The volumes of the proximal part and distal part were calculated as the sum of the volume of each segment of the proximal and distal range [11]:

$$
\mathrm{V}=\mathrm{h}\left(\mathrm{Cl}^{2}-\mathrm{C} 1 \mathrm{C} 2-\mathrm{C}^{2}\right) / 12 \pi
$$

where $\mathrm{V}$ is the volume of the segment, $\mathrm{C} 1$ and $\mathrm{C} 2$ are the circumferences at the end of each segment, and $h$ is the distance (segment length) between $\mathrm{C} 1$ and $\mathrm{C} 2$.

\section{Functional status}

To assess the functional status of patients, we used the Disability of the Arm, Shoulder, and Hand (DASH) questionnaire by the Institute for Work \& Health in 2006. The DASH consisted of 30 items related to physical functionality and symptoms. The score was calculated as:

$$
\text { DASH score }=(\text { sum of scores } / \mathrm{n}-1) \times 25
$$

where $\mathrm{n}$ is the number of items answered. Patient DASH scores were assessed before treatment and 2 weeks after treatment.

\section{Treatment satisfaction}

Treatment satisfaction was assessed by a survey administered after treatment wherein a visual analog scale with a total of 10 items rated 0 to 10 points was used. The questionnaire consisted of items, such as the degree of unwinding after wrapping the bandage, discomfort after wrapping the bandage, difficulty in wrapping the bandage, inconvenience in performing physical activity after 
bandage wrapping, and the effect on daily life after wrapping the bandage. The total score was 100 points. A lower score was indicative of a more difficult and uncomfortable treatment (Supplementary Fig. S1).

\section{Statistical analyses}

The number of subjects was calculated using the G*Power version 3.1 (http://www.psychologie.hhu.de/ arbeitsgruppen/allgemeine-psychologie-und-arbeitspsychologie/gpower.html). The number of samples was maintained at a significance level of 0.05 in the t-test, and a total of 42 people were required. A total of $46 \mathrm{sub-}$ jects were selected to account of dropping out of participants.

The subjects were randomized into the spiral or spica groups by an allocation sequence generated from a computerized random number table. Twenty-three patients were assigned to each group according to a random number table, prior to treatment.

Student's t-tests were used to compare parameters between the two different bandage method groups using
SPSS version 25.0 (IBM Corp., Armonk, NY, USA). The significance level was set at $\mathrm{p}<0.05$. Parameters compared included age, BMI, QOL score, onset after surgery, disease period, difference in volume of the affected limbs, DASH scores before and after treatment, and treatment satisfaction after 2 weeks of CDT. A chi-square test was used to assess the correlations among breast cancer stage, type of surgery, and type of lymph node surgery.

\section{RESULTS}

A total of 46 patients were recruited for this study. Two patients in each group failed to complete the evaluation due to failure to complete the survey or treatment. Therefore, we assessed data collected from 42 patients.

The demographics of each group are shown in Table 1 . There were no statistically significant differences between the two groups in terms of sex, BMI, breast cancer stage, the time between surgery to the onset of lymphedema, disease period, QOL score, type of surgery, type of lymph node surgery, and the volume of the upper limbs with

Table 1. Lymphedema patient characteristics

\begin{tabular}{|c|c|c|c|}
\hline Variable & Spiral method $(n=21)$ & Spica method $(n=21)$ & p-value \\
\hline Age (yr) & $57.3 \pm 56.4(39-70)$ & $56.4 \pm 7.9(42-82)$ & 0.780 \\
\hline $\operatorname{BMI}\left(\mathrm{kg} / \mathrm{m}^{2}\right)$ & $28.0 \pm 8.3$ & $26.1 \pm 2.6$ & 0.576 \\
\hline QOL score & $279.4 \pm 45.5$ & $289.9 \pm 36.6$ & 0.604 \\
\hline Onset after surgery (mo) & $37.8 \pm 45.2$ & $27.4 \pm 36.7$ & 0.429 \\
\hline Disease period (mo) & $6.8 \pm 7.5$ & $8.4 \pm 12.4$ & 0.613 \\
\hline Breast cancer stage & & & 0.148 \\
\hline Stage I & 8 & 7 & \\
\hline Stage II & 2 & 7 & \\
\hline Stage III & 6 & 3 & \\
\hline Unknown & 5 & 4 & \\
\hline Type of surgery & & & 0.799 \\
\hline Mastectomy & 4 & 5 & \\
\hline BCS & 8 & 6 & \\
\hline MRM & 9 & 10 & \\
\hline Type of lymph node surgery & & & 0.929 \\
\hline ALND & 8 & 7 & \\
\hline SLND & 1 & 1 & \\
\hline
\end{tabular}

Values are presented as mean \pm standard deviation (range).

Total volume was measured by volumetry. Volume (below \& above elbow) was calculated by the circumference of the arm. QOL score is the sum of the scores obtained by the patient in The Quality of Life Instrument.

BMI, body mass index; BCS, breast-conserving surgery; MRM, modified radical mastectomy; ALND, axillary lymph node dissection; SLND, sentinel lymph node dissection. 
Table 2. Change from pre- to post-treatment for outcome variables

\begin{tabular}{|c|c|c|c|}
\hline Variable & Spiral method $(n=21)$ & Spica method $(n=21)$ & p-value \\
\hline \multicolumn{4}{|l|}{ Before CDT } \\
\hline Total volume $(\mathrm{mL})$ & $2,149.2 \pm 719.9$ & $2,072.9 \pm 616.1$ & 0.815 \\
\hline Volume, below elbow (mL) & $926.9 \pm 308.1$ & $931.0 \pm 258.4$ & 0.962 \\
\hline Volume, above elbow (mL) & $1,038.7 \pm 359.3$ & $1,085.6 \pm 301.2$ & 0.962 \\
\hline DASH score & $32.8 \pm 19.7$ & $40.3 \pm 11.7$ & 0.150 \\
\hline \multicolumn{4}{|l|}{ After CDT } \\
\hline Total volume (mL) & $2,051.2 \pm 158.3$ & $1,873.9 \pm 125.1$ & 0.589 \\
\hline Volume, below elbow (mL) & $869.0 \pm 261.4$ & $853.9 \pm 217.5$ & 0.826 \\
\hline Volume, above elbow (mL) & $982.7 \pm 292.8$ & $979.4 \pm 243.7$ & 0.826 \\
\hline DASH score & $29.5 \pm 17.3$ & $31.0 \pm 13.9$ & 0.753 \\
\hline Treatment satisfaction & $53.2 \pm 18.0$ & $50.0 \pm 21.0$ & 0.608 \\
\hline \multicolumn{4}{|c|}{ Difference between before and after CDT } \\
\hline Total volume $(\mathrm{mL})$ & $98.0 \pm 158.3$ & $199.0 \pm 125.1$ & $<0.05^{*}$ \\
\hline Volume, below elbow (mL) & $57.9 \pm 42.7$ & $77.1 \pm 43.7$ & 0.218 \\
\hline Volume, above elbow (mL) & $56.0 \pm 65.4$ & $106.2 \pm 82.2$ & $<0.05^{*}$ \\
\hline DASH score & $3.8 \pm 5.4$ & $7.7 \pm 6.6$ & $<0.05^{*}$ \\
\hline
\end{tabular}

Values are presented as mean \pm standard deviation.

Total volume was measured by volumetry. Volume (below \& above elbow) was calculated by the circumference of the arm.

CDT, complex decongestive therapy; DASH, Disability of the Arm, Shoulder, and Hand.

${ }^{*} \mathrm{p}<0.05$.

lymphedema (Tables 1, 2).

When comparing the treatment outcomes for the spiral method and spica method groups, the total volume reduction after treatment was $98.0 \pm 158.3 \mathrm{~mL}$ in the spiral method group and $199.0 \pm 125.1 \mathrm{~mL}$ in the spica method group. Therefore, the spica method group showed a significantly better improvement $(\mathrm{p}<0.05)$.

The circumference was measured at intervals of $3 \mathrm{~cm}$ to calculate the volume of the distal and proximal parts of the affected limb. Improvement was observed in the distal part in the spiral method group and in the distal part in the spica method group. The volume difference in the proximal part before and after treatment was 56.0 \pm 65.4 $\mathrm{mL}$ for the spiral method and $106.2 \pm 82.2 \mathrm{~mL}$ for the spica method, representing a significantly greater reduction in volume for patients in the spica group compared to that for patients in the spiral group ( $\mathrm{p}<0.05)$ (Table 2, Fig. 2).

DASH score changes following the treatment showed that the spiral group score increased by $3.8 \pm 5.4$ and the spica group score increased by $7.7 \pm 6.1$, which was a significantly better improvement $(\mathrm{p}<0.05)$. Treatment satisfaction, which measured patient comfort and compliance while wearing the bandage, showed a higher score with spiral treatment, but this was not statistically significant.

Finally, neither group demonstrated a significant difference in volume reduction between the proximal part and the distal part following treatment (Table 3, Fig. 3).

\section{DISCUSSION}

Previous studies have reported BCRL in $6 \%$ to $83 \%$ of surgical cases [12]. Various methods such as sequential limb circumference, water displacement volume, lymphoscintigraphy, BIA, ultrasound, and tissue tonometry are used to diagnose lymphedema. In a recent study, there was a discussion that increased FDP and D-dimer were associated with lymphedema [13]. In this study, lymphoscintigraphy and BIA were used to diagnose lymphedema.

Lymphoscintigraphy is a method of imaging the anatomic and transport capacity abnormalities of lymphatic flow. Diagnostic accuracy of lymphedema showed moderate sensitivity $(=0.62)$ and high specificity $(=1.0)$ when compared to unaffected extremities [14]. Additionally, 

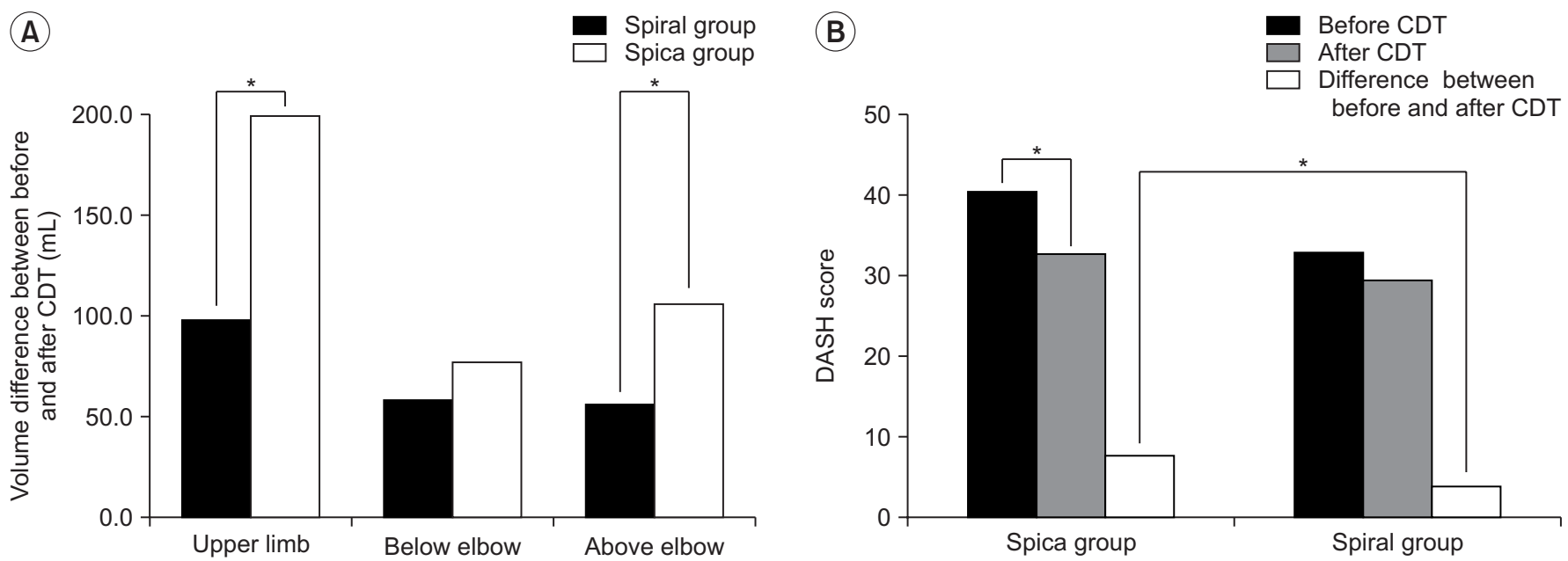

Fig. 2. Differences in variables between before and after CDT in patients with breast cancer related lymphedema. CDT, complex decongestive therapy; DASH, Disability of the Arm, Shoulder, and Hand. * $\mathrm{p}<0.05$.

Table 3. Differences between upper arm and forearm following treatment

\begin{tabular}{lccc}
\hline & $\begin{array}{c}\text { Volume, below } \\
\text { elbow (mL) }\end{array}$ & $\begin{array}{c}\text { Volume, above } \\
\text { elbow (mL) }\end{array}$ & p-value \\
\hline $\begin{array}{c}\text { Spiral method } \\
\text { (n=21) }\end{array}$ & $57.9 \pm 42.7$ & $56.0 \pm 65.4$ & 0.726 \\
$\begin{array}{l}\text { Spica method } \\
(\mathrm{n}=21)\end{array}$ & $77.1 \pm 43.7$ & $106.2 \pm 82.2$ & 0.551 \\
\hline
\end{tabular}

Values are presented as mean \pm standard deviation.

${ }^{*} \mathrm{p}<0.05$.

quantitative lymphoscintigraphic analysis has been found to be associated with the possibility of lymphedema development and can be used as a predictor of the likelihood of edema after surgery [15]. However, there are disadvantages of high costs, low resolution, invasiveness, and radiation exposure.

The bioimpedance measurement is a method to calculate the amount of body fluid by measuring the impedance to the current passing through the body part. Previous studies have shown that the treatment effects can be predicted by the ratio of extracellular fluid volume and single frequency BIA to the affected limb and unaffected limb. BIA can be used as a useful indicator to predict the treatment outcome [16].

Lymphedema can be treated with medication or surgery; however, CDT has recently been adopted as the typical treatment method for patients with lymphedema [17]. CDT involves non-elastic compression bandages, MLD, compression garments, exercise, and self-care. Among

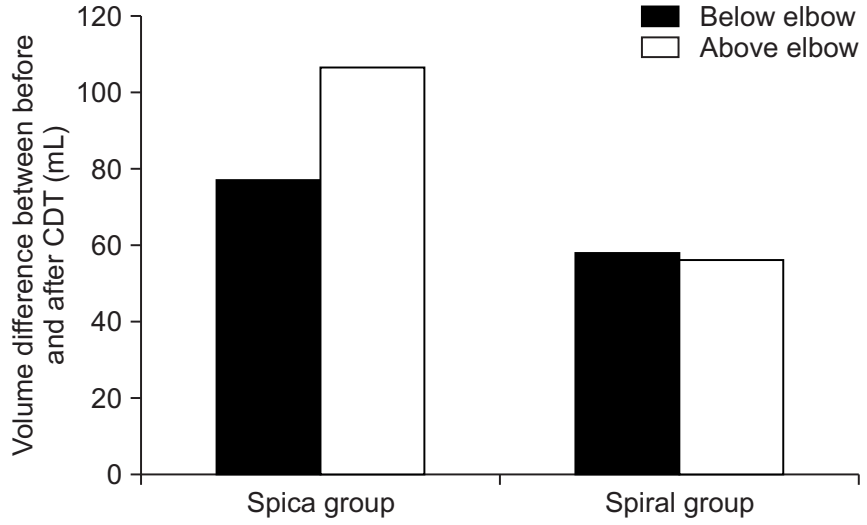

Fig. 3. Volume differences between upper arm and forearm following complex decongestive therapy (CDT).

these various components of the treatment, bandaging plays an important role $[18,19]$.

A variety of materials can be used for bandaging, and it is common to determine if elastic or non-elastic compression bandages should be used with in vitro evaluation. Elasticity is defined as the ratio of elongation when applying a force of $10 \mathrm{~N} / \mathrm{cm}$ bandage width to the material. When the elongation is $0 \%-10 \%$, between $10 \%$ to $100 \%$, and $100 \%$, then the bandage is considered a 'no stretch' bandage, short stretch bandage, and long stretch bandage, respectively [20]. Additionally, on applying multiple layers of elastic bandages, the same effect can be achieved as that achieved on the use of non-elastic bandages. This non-elastic multilayer bandaging method is the most commonly used method for the early treat- 
ment of lymphedema [21].

Most studies on the effects of CDT and bandaging have used the spiral bandaging technique [22]. However, the spiral method, which involves bandaging the patient's arm in one direction, is not the only available method; the spica method, which involves application of a bandage in a zigzag manner, can also be used.

Spica bandaging is a method that has been mainly used when it is necessary to perform bandaging of areas where the bandage may be easily loosened, such as the hip. One study reported that bandaging using the spica method for lower extremity edema patients demonstrated better reduction of edema [23]. However, this is the first study to compare the effects of these two methods in patients with upper limb edema after breast cancer surgery.

Previous studies of patients with lymphedema that assessed the International Classification of Disease, Disability, and Health (ICF) demonstrated that lymphatic edema adversely affects most body functions, body structures, activities, and participation $[24,25]$. In another study, combining resistance exercise and intensive CDT for lymphedema had a positive effect on the incidence of exacerbations, the severity of lymphedema symptoms, arm disabilities, muscle strength, and also indicated an improved DASH score [26]. In this study, the DASH score was evaluated before and after treatment to compare the degree of improvement for each bandage method in addition to the degree of edema reduction. A survey was also conducted after treatment to compare patient difficulty, discomfort, and compliance with the different bandaging methods.

We report that the spica group had a significantly greater decrease in the volume of the upper limb (especially above the elbow) compared to the spiral group, and the spica group also had a significantly greater improvement in the DASH score. The spiral method demonstrated higher patient satisfaction scores than those for the spica method, but this difference was not significant.

However, there is difficulty in that the spica method should be zigzagged compared to the spiral method, which can simply be wound in one direction. Although this may be considered simple, the small difference may cause difficulty in performing the bandage technique because only one arm should be used to bandage in the opposite arm with lymphedema. It takes a long time to wrap the bandage and is more difficult for the patient to learn. Moreover, since the spica method is more firmly fixed to the proximal part of the affected limb, the patient may feel tightness due to the nature of the bandage to be maintained over a long period of time. These disadvantages can reduce patient compliance.

Bandages as a component of CDT are clinically effective at reducing edema in the distal part of the upper extremity affected by lymphedema, and resistive exercise is more effective at reducing edema in the proximal part. Additionally, previous studies have shown that active resistive exercise in patients with BCRL results in better outcomes for the proximal part of the affected upper extremity than CDT alone [2]. Importantly, this study demonstrates that the use of a different bandaging method, without additional exercise therapy, can increase therapeutic effects on the proximal part of the upper extremity with lymphedema, which can be called the limit of bandaging therapy.

CDT is divided into two stages. The first stage of treatment minimizes limb volume through skin care, MLD, physical exercises, and non-elastic compression bandages. The second stage or maintenance stage is performed immediately after the first stage and involves the application of elastic compression stockings, exercises, and selfmassage to maintain and optimize the results obtained in the first stage [27].

Bandages, which play an important role in the first stage of lymphedema treatment, can be difficult to apply properly due to loosening of the bandage. Various alternative methods have been devised to overcome this drawback, including the use of non-elastic Velcro or wrapping the limb with a wrap [28]. However, the spica bandaging method used in our study was able to prevent bandage loosening without the use of these alternative strategies and was more effective at reducing the volume of the upper extremity than the conventional spiral method, particularly for the proximal part of the limb.

In summary, the purpose of this study was to compare the treatment efficacy and patient satisfaction following bandage techniques using the spiral method and the spica method. The treatment efficacy was evaluated as the reduction of volume of the upper limb with edema after treatment, and the DASH score was used to evaluate the improvement of the patient's performance ability. Additionally, satisfaction with and discomfort of the treatments were assessed using a patient satisfaction ques- 
tionnaire. The results demonstrate that the spica method was better able to reduce edema and enhance function compared to the spiral method.

According to the International Society of Lymphology, lymphedema can be divided into three stages. Stage 0 is defined by subtle changes in the tissue fluid and composition and subjective symptoms. In stages I-III, clear swelling occurs and can last for months or years [29]. However, we did not classify the patients' lymphedema stage in this study. We were unable to determine the differences in the effects of bandaging method based on the stage. Therefore, further research will be required to better assess the effects of different bandaging methods in patients with different stages of lymphedema.

In conclusion, the purpose of this study was to compare the therapeutic effects of different bandage methods performed as a component of CDT, an important method for the treatment of lymphedema. We compared outcomes following two methods of bandaging: the spiral method and the spica method. The spica method demonstrated better volume reduction and DASH score improvement than the spiral method. However, the spiral method showed a higher score for patient satisfaction, but this was not statistically significant. In conclusion, our results suggest that the spica method may be more effective for treating patients with lymphedema, but it may be more difficult and uncomfortable for the patient. Therefore, the treatment method should be selected according to the degree of symptoms and patient compliance. Finally, the spica method is recommended for intensive care situations.

\section{CONFLICT OF INTEREST}

No potential conflict of interest relevant to this article was reported.

\section{AUTHOR CONTRIBUTION}

Conceptualization: Oh SH, Sim YJ. Methodology: Sim YJ, Jeong HJ, Lee JH. Formal analysis: Oh SH, Ryu SH. Funding acquisition: none. Project administration: Sim YJ, Jeong HJ, Lee JH. Visualization: Oh SH, Ryu SH. Writing - original draft: Oh SH, Ryu SH. Writing - review and editing: all authors. Approval of final manuscript: all authors.

\section{SUPPLEMENTARY MATERIALS}

Supplementary materials can be found via https://doi. org/10.5535/arm.2019.43.6.677. Fig. S1. Bandage Therapy Satisfaction Questionnaire.

\section{REFERENCES}

1. Kim SJ, Yi CH, Kwon OY. Effect of complex decongestive therapy on edema and the quality of life in breast cancer patients with unilateral leymphedema. Lymphology 2007;40:143-51.

2. Kim DS, Sim YJ, Jeong HJ, Kim GC. Effect of active resistive exercise on breast cancer-related lymphedema: a randomized controlled trial. Arch Phys Med Rehabil 2010;91:1844-8.

3. King M, Deveaux A, White H, Rayson D. Compression garments versus compression bandaging in decongestive lymphatic therapy for breast cancer-related lymphedema: a randomized controlled trial. Support Care Cancer 2012;20:1031-6.

4. Tsai HJ, Hung HC, Yang JL, Huang CS, Tsauo JY. Could Kinesio tape replace the bandage in decongestive lymphatic therapy for breast-cancer-related lymphedema? A pilot study. Support Care Cancer 2009;17: 1353-60.

5. Kligman L, Wong RK, Johnston M, Laetsch NS. The treatment of lymphedema related to breast cancer: a systematic review and evidence summary. Support Care Cancer 2004;12:421-31.

6. Karki A, Anttila H, Tasmuth T, Rautakorpi UM. Lymphoedema therapy in breast cancer patients: a systematic review on effectiveness and a survey of current practices and costs in Finland. Acta Oncol 2009; 48:850-9.

7. Badger C, Preston N, Seers K, Mortimer P. Physical therapies for reducing and controlling lymphoedema of the limbs. Cochrane Database Syst Rev 2004;(4): CD003141.

8. Armer JM, Stewart BR. A comparison of four diagnostic criteria for lymphedema in a post-breast cancer population. Lymphat Res Biol 2005;3:208-17.

9. Ridner SH. Quality of life and a symptom cluster associated with breast cancer treatment-related lymphedema. Support Care Cancer 2005;13:904-11.

10. Zuther JE. Pathology: documentation techniques for 
lymphedema. In: Zuther JE, editor. Lymphedema management: the comprehensive guide for practitioners. 2nd ed. New York: Thieme; 2005. p. 127-9.

11. Taylor R, Jayasinghe UW, Koelmeyer L, Ung O, Boyages J. Reliability and validity of arm volume measurements for assessment of lymphedema. Phys Ther 2006;86:205-14.

12. Clark B, Sitzia J, Harlow W. Incidence and risk of arm oedema following treatment for breast cancer: a threeyear follow-up study. QJM 2005;98:343-8.

13. Ryu SH, Min SW, Kim JH, Jeong HJ, Kim GC, Kim DK, et al. Diagnostic significance of fibrin degradation products and D-dimer in patients with breast cancerrelated lymphedema. Ann Rehabil Med 2019;43:81-6.

14. Levenhagen K, Davies C, Perdomo M, Ryans K, Gilchrist L. Diagnosis of upper quadrant lymphedema secondary to cancer: clinical practice guideline from the oncology section of the American Physical Therapy Association. Phys Ther 2017;97:729-45.

15. Kim P, Lee JK, Lim OK, Park HK, Park KD. Quantitative lymphoscintigraphy to predict the possibility of lymphedema development after breast cancer surgery: retrospective clinical study. Ann Rehabil Med 2017;41:1065-75.

16. Kim L, Jeon JY, Sung IY, Jeong SY, Do JH, Kim HJ. Prediction of treatment outcome with bioimpedance measurements in breast cancer related lymphedema patients. Ann Rehabil Med 2011;35:687-93.

17. Didem K, Ufuk YS, Serdar S, Zumre A. The comparison of two different physiotherapy methods in treatment of lymphedema after breast surgery. Breast Cancer Res Treat 2005;93:49-54.

18. Casley-Smith JR, Casley-Smith JR. Modern treatment of lymphoedema. I. Complex physical therapy: the first 200 Australian limbs. Australas J Dermatol 1992; 33:61-8.

19. Matthews K, Smith J. Effectiveness of modified complex physical therapy for lymphoedema treatment. Aust J Physiother 1996;42:323-8.

20. Damstra RJ, Partsch H. Compression therapy in breast cancer-related lymphedema: a randomized, con- trolled comparative study of relation between volume and interface pressure changes. J Vasc Surg 2009;49: 1256-63.

21. Partsch H, Clark M, Mosti G, Steinlechner E, Schuren J, Abel M, et al. Classification of compression bandages: practical aspects. Dermatol Surg 2008;34:600-9.

22. Mayrovitz HN. The standard of care for lymphedema: current concepts and physiological considerations. Lymphat Res Biol 2009;7:101-8.

23. Benigni JP, Uhl JF, Cornu-Thenard A, Blin E. Compression bandages: influence of techniques of use on their clinical efficiency and tolerance. Int Angiol 2008;27: 68-73.

24. Devoogdt N, De Groef A, Hendrickx A, Damstra R, Christiaansen A, Geraerts I, et al. Lymphoedema Functioning, Disability and Health Questionnaire for Lower Limb Lymphoedema (Lymph-ICF-LL): reliability and validity. Phys Ther 2014;94:705-21.

25. Viehoff PB, Gielink PD, Damstra RJ, Heerkens YF, van Ravensberg DC, Neumann MH. Functioning in lymphedema from the patients' perspective using the International Classification of Functioning, Disability and health (ICF) as a reference. Acta Oncol 2015;54:411-21.

26. Do JH, Kim W, Cho YK, Lee J, Song EJ, Chun YM, et al. Effects of resistance exercises and complex decongestive therapy on arm function and muscular strength in breast cancer related lymphedema. Lymphology 2015; 48:184-96.

27. Ezzo J, Manheimer E, McNeely ML, Howell DM, Weiss $\mathrm{R}$, Johansson KI, et al. Manual lymphatic drainage for lymphedema following breast cancer treatment. Cochrane Database Syst Rev 2015;(5):CD003475.

28. Campanholi LL, Lopes GC, Mansani FP, Bergmann A, Baiocch JM. The validity of an adjustable compression Velcro wrap for the treatment of patients with upper limb lymphedema secondary to breast cancer: a pilot study. Mastology 2017;27:206-12.

29. International Society of Lymphology. The diagnosis and treatment of peripheral lymphedema: 2013 Consensus Document of the International Society of Lymphology. Lymphology 2013;46:1-11. 